\title{
A MOST open-field data reduction software and its applications to BRITE
}

\author{
D. Huber ${ }^{1}$, P. Reegen ${ }^{1}$ \\ ${ }^{1}$ Institut für Astronomie, Türkenschanzstrasse 17, 1180 Vienna, Austria
}

\section{Abstract}

A data reduction pipeline specifically developed for space-based open-field photometry obtained with the MOST satellite is presented. The reduction steps include correction of cosmic ray events, identification and rejection of exposures showing distorted images due to pointing instabilities and efficient correction of stray light influences on the CCD. Considering the planned instrumentation for the BRITE mission, suggestions for an efficient data reduction based on the experiences with MOST are made. It is concluded that under the current specifications for the mission many of the developed techniques might be directly applicable for the development of a BRITE data reduction pipeline.

\section{Introduction}

The MOST (Microvariability and Oscillations of STars) space mission (Walker et al. 2003) was launched in June 2003 to perform high-precision space photometry of bright stars. Equipped with a $15 \mathrm{~cm}$ Rumak-Maksutov telescope and a custom broadband filter $(350-700 \mathrm{~nm})$, MOST is positioned in a polar, sun-synchronous orbit, enabling the satellite to monitor the same star for up to 2 months without interruptions.

In the original design MOST was equipped with two CCDs, one for science and one for tracking, the latter however being disfunctional due to a particle hit since early 2006. The primary observing mode makes use of a Fabry microlens array. Entering a field stop of 1 arcmin diameter, an extended defocused image of the telescope's entrance pupil is projected on the science CCD. This image is very insensitive to pointing instabilities, hence provides a high photometric quality. Secondary science is performed on the remaining areas of the CCD, corresponding to conventional CCD photometry as will be performed in the BRITE mission. Figure 1 shows a typical point-spread function (PSF) of the MOST open field photometry. The typical FWHM of the MOST PSF is about 2-3 pixels, with one pixel corresponding to 0.3 arcsec. 


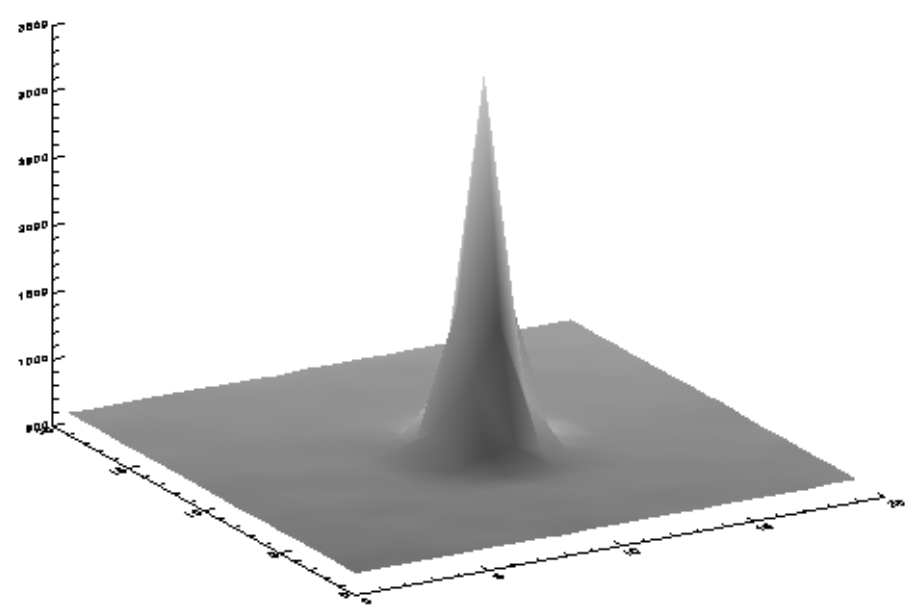

Figure 1: Typical PSF of MOST open field photometry. The FWHM is about 2-3 pixels.

The main data reduction pipeline for the primary Fabry Imaging observing mode is fully described in Reegen et al. (2006). Based on the techniques used for this pipeline, we developed a reduction software for processing open-field photometry which will be presented in detail in the following sections. An independent reduction for this observing mode was also developed by Rowe et al. (2006).

\section{Aperture determination}

Since possible satellite jitter due to pointing instabilities sometimes causes the PSF to wander over the CCD, it is not a priori possible to set a fixed aperture for all exposures of the same observing run. Many reduction steps however are based on a fixed identification of pixels illuminated by starlight (hereafter target pixels) and sky signal (hereafter background pixels) in order to analyze and correct instrumental influences over time rather than locally on one exposure. To realize an aperture determination, a gaussian function of the form

$$
F(x, y)=A_{0}+A_{1} e^{-\frac{U}{2}}
$$

with the elliptical function 


$$
U=\left(\frac{x^{\prime}}{a}\right)^{2}+\left(\frac{y^{\prime}}{b}\right)^{2}
$$

is fitted to each exposure. The ellipse with semi-major axis $a$ and semi-minor axis $b$ is allowed to rotate in the coordinate system $(x, y) \rightarrow\left(x^{\prime}, y^{\prime}\right)$ by applying a rotation matrix in cartesian coordiantes, yielding

$$
\begin{aligned}
& x^{\prime}=(x-h) \cos T-(y-k) \sin T \\
& y^{\prime}=(x-h) \sin T-(y-k) \cos T
\end{aligned}
$$

with $T$ being the rotation angle in clockwise direction of $a$ and $b$ with respect to the $\mathrm{x}$ - and $\mathrm{y}$-axis, and $(h, k)$ denoting the center position of the ellipse. $A_{0}$ denotes a constant offset of the function from the zero point, and $A_{1}$ a scaling factor to unity. A least-squares fit yields the 7 parameters $\left(A_{0}, A_{1}, a, b, h, k, T\right)$ which completely describe the airy disk of the star exposure. In the most straightforward approach, this procedure allows to define target pixels simply as all pixels showing larger intensities than the surface constant $A_{0}$.

\section{Cosmics Correction}

The automatic identification and correction of cosmic ray events constitutes an important part of the data reduction of space based imaging. Especially during the crossing of the South Atlantic Anomaly, cosmic hits can severely influence the count rates and deterior the derived results from the data. For MOST openfield photometry, a two step strategy has been developed to identify cosmics in target and background pixels, respectively.

\section{Background Pixels}

As a first step a determination of target and background pixels as described in the previous section is performed for each exposure. In the case of background pixels, the identification of cosmics is then based on a simple sigma-clipping procedure. The standard deviation $\sigma$ is computed for all background pixels and every pixel intensity $G$ fullfilling the condition

$$
I>k \sigma
$$

is flagged as a cosmic candidate ( $k$ being an integer value, usually set to 4 ). The cosmic candidate is then replaced by the mean value of all surrounding background pixels which were not flagged. Additionally, a limit for the maximum number of cosmic candidates per frame is set. In the default mode this 
limit is set to 10 , i.e. if 10 out of all pixels of the $20 \times 20$ subraster are flagged as cosmic candidates, the exposure is rejected.

\section{Target Pixels}

On the contrary to background pixels, the cosmic identification for target pixels is based on comparing pixel intensities over time rather than locally on the same image. To achieve this, a distant-weighted average (DWA) of the form

$$
F_{\nu}(t)=\frac{\sum_{n=1, t_{n \neq t}}^{N}\left|t-t_{n}\right|^{-\nu} I_{n}}{\sum_{n=1, t_{n \neq t}}^{N}\left|t-t_{n}\right|^{-\nu}}
$$

where $N$ is the number of frames used to average, $I_{n}$ the mean intensity of all target pixels of the frame $n$ and $\nu$ a weighting parameter ( $\nu=0$ corresponds to a normal arithmetic mean) is computed. Essentially, a DWA returns a value averaged and weighted over time, e.g. a datapoint further apart from the value of interest is weighted less. The range over which the DWA is calculated is a free parameter, usually set to $10-20$ frames. Since an anomalous value due to a cosmic ray event does sometimes persist over a couple of images this method is appropriate for detecting such an event. Considering a datapoint at times $n$, with a mean intensity $I_{n}$, a distant weighted average $F_{\nu}\left(\mathrm{t}_{n}\right)$ and a standard deviation of the mean intensities of the DWA range $\sigma$, a cosmic hit is identified if

$$
I_{n}-F_{\nu}\left(t_{n}\right)>k \sigma
$$

is fullfilled, i.e. the deviation of a mean intensity of a frame is large with respect to the distant weighted average mean intensity. The value of $k$ is a free parameter, with a default value of $k=4$. Since all these calculations are based on mean intensities of the stellar image, the only conclusion to be drawn from equation (7) is that there are anomalous pixel values in the target area. Since there is no clean way to recover the actual stellar intensity, each frame showing such a feature is automatically rejected. An example for the application of this procedure to MOST data is shown in Figure 2.

\section{Image Geometry}

In some rare cases, severe pointing instabilities can cause the starlight to enter the telescope aperture with an extreme angle. This results in images showing 

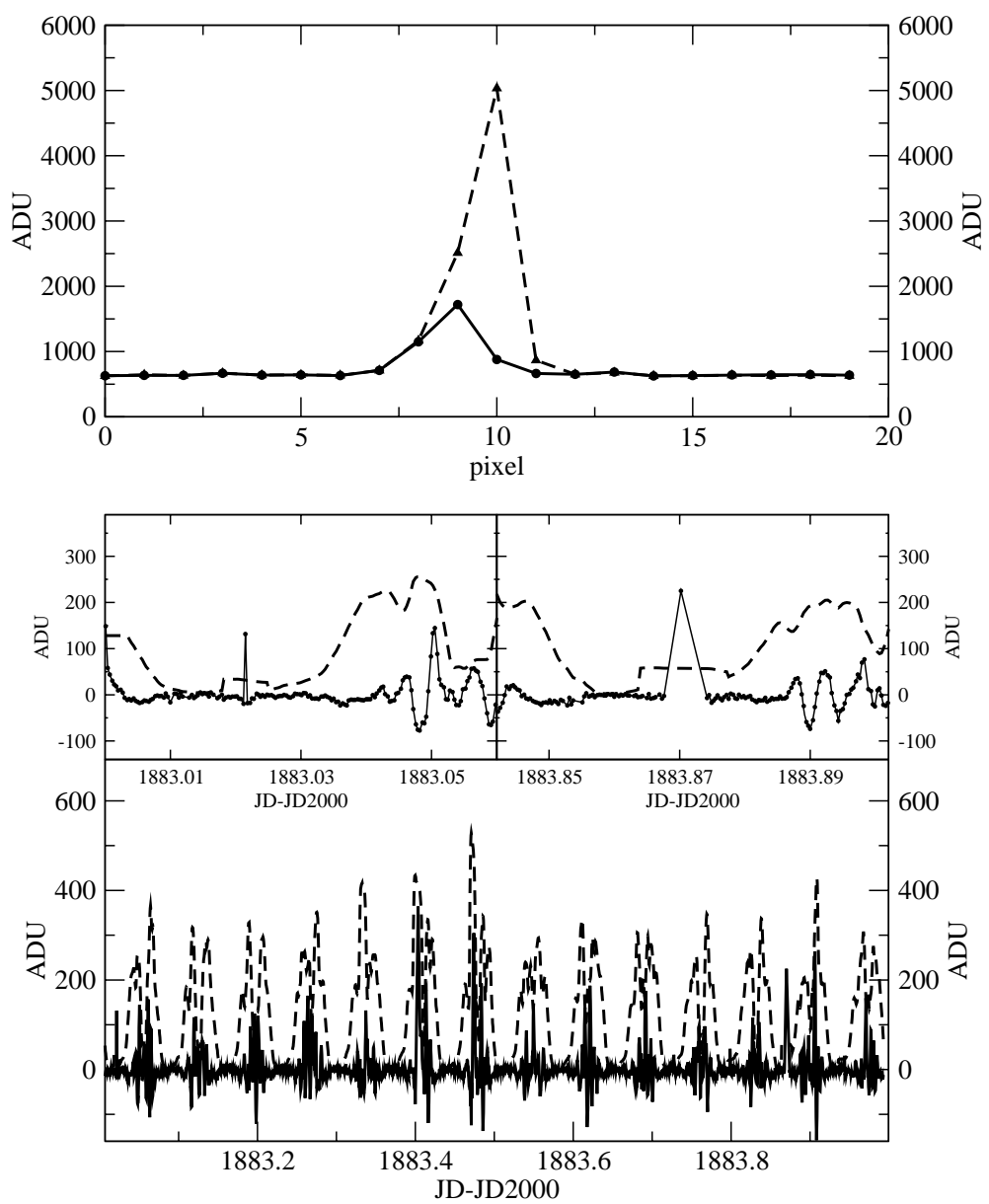

Figure 2: Examples of cosmic ray impacts in the target area of MOST observations. The bottom panel shows the left side of equation (7) (i.e. the deviation of the mean intensity from the calculated DWA, solid line) and a $4 \sigma$ curve of the frames in the DWA range (i.e. right side of equation (7) with $k=4$, dashed line). Two outliers, one at the beginnig and one at the end of the one day dataset, can clearly be identified. The smaller panels show these two areas zoomed in. In the top panel, the dashed line shows the intensity over a row of the $20 \times 20$ subraster (containing the star) for the example of the earlier outlier (JD-JD2000 1883.02). The solid line shows the same plot for the exposure right before the affected image. Clearly, a cosmic ray event has altered the stellar intensity. 
a deformed PSF shape which naturally would lead to disturbed count rates. Figure 3 shows examples for such images in the MOST data. Since there is no way to reconstruct the original count rate, such exposures have to be automatically identified and rejected by the pipeline.
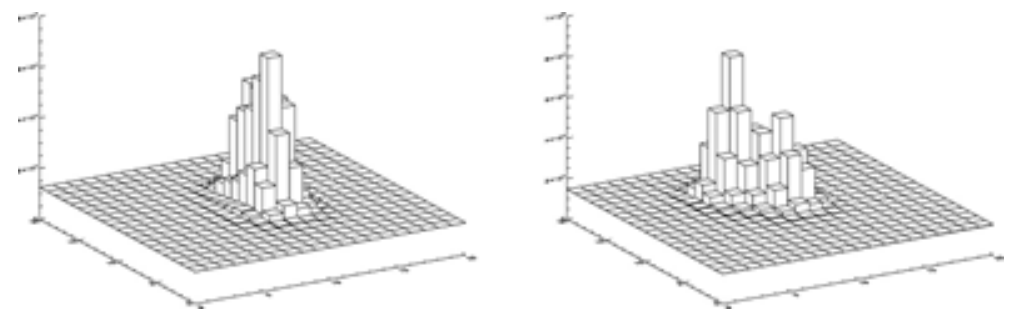

Figure 3: Examples for deformed image geometry due to severe pointing instabilities in MOST data.

To do so, the PSF fitting procedure described is again evaluated. Possible rejection criteria include:

- The fit does not converge.

- The number of target pixels exceeds a certain limit (for a $20 \times 20$ subraster $\sim 150$ ).

- The PSF center coordinates deviate more than a certain limit from the most common center position.

The last criterium is imposed as a preparation for the stray light reduction module, which will be the subject of the following section.

\section{Stray Light Correction}

\section{General Concept and Implementation}

Various influences which are aforehand hard to estimate might cause scattered light from other sources than the star illuminating the CCD. Most prominent examples for this are light from the illuminated side of the Earth or moonshine. As BRITE will most likely not be launched in a sun-synchronous orbit, it is expected that the observations will also be affected by this influence to some extent.

A proper reduction of stray light has been the major challenge in MOST data processing. The technique which was finally developed is based on calculating correlations of target with background intensities over time. An example for 
such an intensity-intensity correlation is shown in Figure 4. Since the influences from stray light are the same for target and background pixels, a correction of this correlation (i.e., a decorrelation) using a linear regression will correct the instrumental signal in the data.

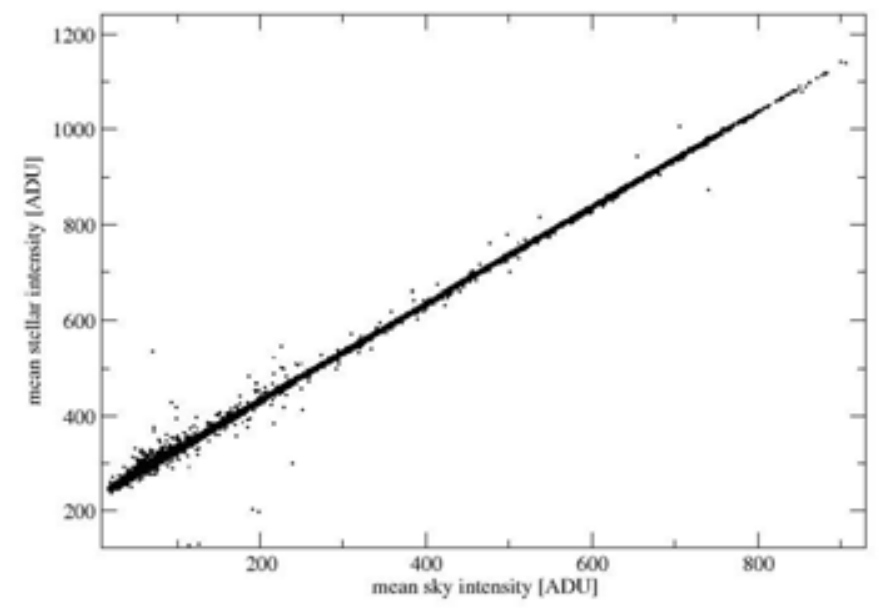

Figure 4: Example for an intensity-intensity correlation diagram for a timespan of several MOST orbital periods used for correcting stray light influences. In this case, mean stellar and sky intensities are plotted.

In the most simple approach, the decorrelation can be performed for mean intensities of target and background pixels, respectively. In reality, however, the stray light pattern across the CCD might not be uniform and show a much more complex structure. For this case, a stepwise pixel-to-pixel decorrelation has been developed (originally for Fabry Imaging, see Reegen et al. 2006). In this procedure, the background pixel showing the best correlation with the mean target intensity is calculated. Consequently, all pixels on the CCD are decorrelated with this pixel. This procedure is repeated with different background pixels until all stray light influences are reduced to a satisfactory extent.

While in the Fabry Imaging mode the extended defocused stellar image remains stable on the CCD, considerable PSF wander can ocurr in the openfield photometry due to minor pointing instabilities. It is obvious that such a variation of the PSF position will prevent a pixel-to-pixel decorrelation, since 
one pixel can change over time from being identified as target or background pixel. To still be able to make use of this powerful technique, an additional routine which aligns the PSF positions had to be implemented to circumvent this problem.

The first difficulty is to be able to define a symmetric aperture around the stellar signal on an individual frame. It is not (or almost never) given that the PSF fits symmetrically within these physical restrictions. Instead, stellar intensity will occupy different amounts of pixel space at each edge. One way to solve this problem is to define a circular aperture on non-integer coordinates and estimate the intensity on a subpixel scale. This can however not be applied here, since the decorrelation method demands whole pixel intensitiy values to be correlated. Another method therefore is to simply transform the point of the PSF defining the symmetry center, i.e. the centroid position, from its original (non-integer) position to the exact center of its pixel. By applying this transformation to all sourrounding pixels as well, the PSF will align much better on integer pixel coordinates, and hence an aperture based on integer pixels will fit more symmetrically. The transformation is done by a bilinear subpixel intensity interpolation applied to all pixels with respect to the centroid position. Having all intensities aligned to a common pixel grid, the final shifting procedure is implemented. Using the centroid position calculated in the step before (using only pixels within the PSF), the integer part of this value is taken and the difference to the mean position (already calculated in an earlier step of the reduction) is determined. This is done in $x$ - and $y$-axis and all intensities are subsequently shifted by this amount of integer pixel numbers. This results in images which are are aligned to the most common PSF position (therefore minimizing the amount of shifting). With these two steps combined, it is now possible to define an aperture symmetrically surrounding the PSF which at the same time fits all images due to their aligned position. It is noted that due to the fact that the shifting can ocurr for different images in different directions, the overlapping common image area will be smaller than before the shifting procedure. Hence, the final image size will be shrinked. An example for the image shifting process for consecutive exposures is shown in Figure 5.

With all images aligned, decorrelation can be applied. Since the stray light is also modulated on long timescales, the correction is performed in subsets considerably smaller than the total observation time. Moreover, to prevent intensity differences between consecutive parts of the light curve, the decorrelation is calculated for a larger subset (typically 5 MOST orbits or $\sim 0.3$ days), but applied only to a smaller subset (typically 0.005 days). The next subset step is then shifted by the small subset size until the end of the dataset is reached. 

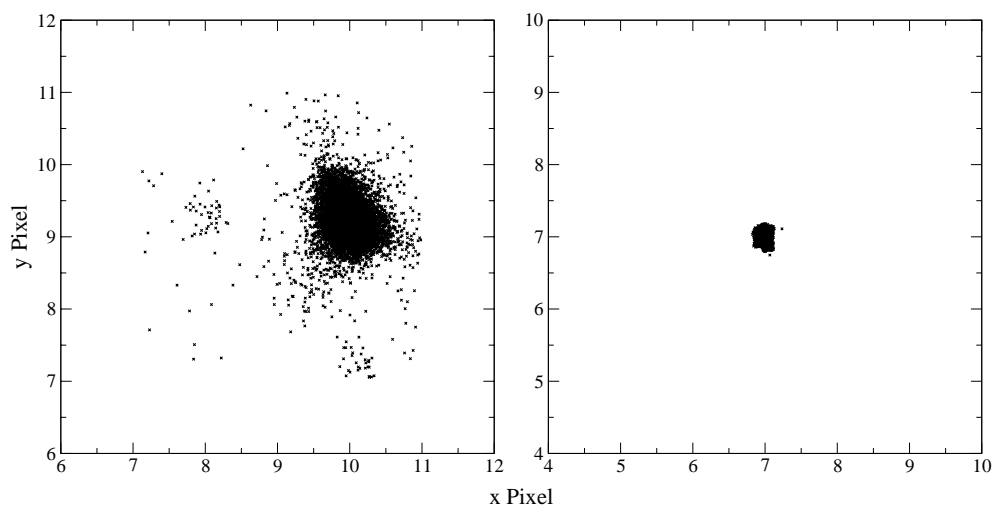

Figure 5: Demonstration of image shifting routine for about 9000 consecutive frames of a MOST run. Each point shows the center coordinates of the calculated PSF for a single exposure. Left: raw images before alignment; Right: processed images after shfting routine. Note that in both cases the axis scales are the same ( $6 \times 6$ pixels).

\section{Restrictions}

There are restrictions in open field photometry which limit the performance of the decorrelation technique compared MOSTs Fabry Imaging mode. Generally, the correlation between pixel intensities seems to be poorer due to the fact that the star light is focused on only a few pixels rather than spread out evenly over the CCD as is the case for Fabry Imaging. If a considerable stray light gradient over the CCD subraster of interest is present, this significantly limits the efficiency of pixel-to-pixel decorrelation. Therefore in some cases, this approach can introduce significant scatter in the reduced light curve. The reduction software therefore allows to choose between using mean intensities and/or pixel intensities for decorrelation.

Another important aspect concerning observation strategy is image stacking, which has been implemented for the MOST mission after the loss of the startracker CCD in early 2006. It has been found that stacking intensities over longer timescales significantly worsens correlations of star and background intensities. This is most likely due to the fact that the stray light is variable on timescales shorter than the total exposure time of the combined subexposures, which smears out the stray light effects over time. Hence, stray light influences appearing in target and background pixels will deviate and cause non-linearities in the intensity-intensity diagrams. To compensate for this problem, higher order fits have been implemented to correct the correlation. Additionally, an outlier rejection routine was included to improve the correlations. Nevertheless, 
image stacking is still a persistent problem and slightly limits the effectiveness of the stray light correction technique.

\section{Dark \& Flatfield Calibration}

Due to the limited budget of small space telescope missions such as MOST or BRITE, no or few moveable parts can be realized when building the satellite. This prevents the ability to perform standard CCD reduction procedures such as dark current measurements or flatfield calibrations.

For the reduction of MOST photometry, however, Rowe et al. 2006 proposed an alternative approach to obtain such measurements without using a mechanical shutter. Using exposures during high stray light phases, CCD subraster regions which are free of light from sourrounding stars are recovered. After removing the gradient caused by the stray light with a polynomial fit, the resulting images can be used as flatfield and dark frames, respectively. Due to lack of testing time, this routine has not yet been implemented in the reduction software presented here. For more information, we refer to Rowe et al. (2006).

\section{An Example}

Figure 6 shows an example of the application of the data reduction to MOST photometry. The top panel, showing the raw data extracted by simple aperture photometry, demonstrates the complexity of the stray light behavior. Besides the main intensity peaks reappearing with the orbital period of the satellite $(\sim$ $101 \mathrm{~min}$ ), a longer modulation trend of the stray light can be identified. The bottom panel of the same plot shows the light curve after the reduction has been completed. The running average indicated by the solid line shows the clear intrinsic variability of the star which could only be guessed in the raw light curve. Indeed, this star is a $\delta$ Scuti pulsator discovered by MOST.

\section{Applications for BRITE}

Almost all techniques described above do not depend specifically on the characteristics of MOST and should therefore be easily applicable for other high-duty cycle CCD photometry. An important aspect however is the combination of PSF fitting and aperture photometry used which naturally critically depends on the shape of the PSF.

For BRITE, the current design forsees a largely defocused PSF (4-5 pixels FHWM) which actually favors the application of the decorrelation technique for stray light correction. However, the defocused PSF is currently planned to 


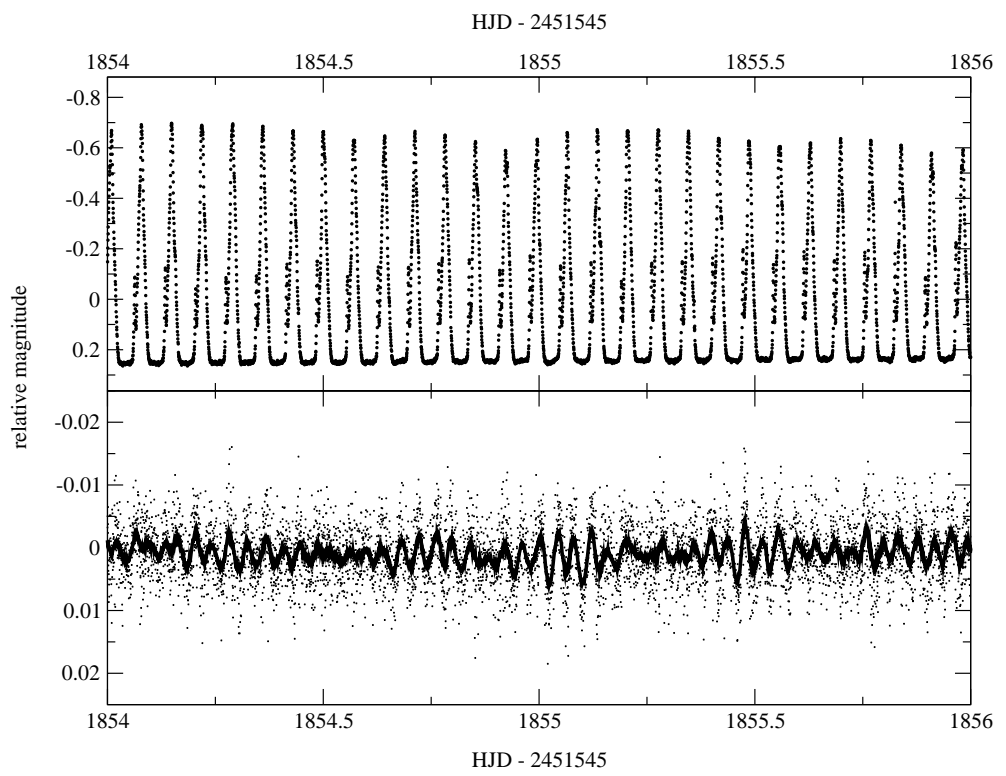

Figure 6: Subset of MOST photometry of a $\delta$ Scuti pulsator spanning over two days. The top panel shows the raw data, the bottom panel the reduced light curve using the techniques described above. The solid line in the bottom panel shows a running average of the data, clearly revealing the light variations of the star. Note the difference in $y$-axis scaling between the two panels.

be realized by combining subexposures which due to satellite jitter would cause the stellar light to be distributed more evenly over the CCD. If such an image stacking is realized this way, it will, based on the current experience with MOST data, be extremely important to save information of the background readings in the subexposures at some point. Although the mission is concipated to only observe very bright stars, the exposure times will still most likely be large compared to the variation timescales of possible stray light. Therefore, if no background information is saved it will be very difficult to recover the stray light pattern and therefore the correlation of instrumental artefacts polluting both target and background pixels.

The application of the routines described will largely depend on the ability to model the PSF in the manner it is possible for MOST using a simple 3D gaussian function. The use of a distance weighted average routine to identify outliers based on a temporal rather than a local comparison is a powerful tool for space missions which show regular sampling for a long time of observations. In any case, the principles and lessons learned from MOST for handling the major 
difficulties in space based CCD photometry such as cosmic ray events, influences of pointing instabilities and stray light will most likely be applicable to BRITE in some sense. The development of a detailed application will certainly only become possible when real data become available and the specific characteristics of the photometry are known.

\section{Conclusions}

We conclude that most of the procedures developed for the reduction of spacebased photometry by MOST will most likely be applicable to the BRITE mission. However, important differences will have to be taken into account, especially the differences in the shape of the PSF. Recent MOST photometry has shown that although image stacking does significantly enhance $S / N$, its consequences for correcting possible stray light influences can be quite severe. If possible, a largely defocused PSF spreading the star light as evenly as possible over the CCD without having to resort to image stacking would be the best option for the BRITE mission if a large amount of complex stray light influences is expected. If image stacking is applied, saving information about the background level of the subexposures will be an important option for correcting instrumental influences. In any case, the experience gathered with the wealth of data obtained by MOST will positively enhance (and hopefully speed up) the developed of a reduction pipeline for BRITE.

Acknowledgments. The authors thank Thomas Kallinger, Denis Frast, Werner Weiss and the rest of the Vienna MOST data reduction team for their pioneering work on developing the Fabry Imaging reduction pipeline which served as the base for many applications implemented in the MOST open-field data reduction.

\section{References}

Reegen, P., Kallinger, T., Frast, D., et al. 2006, MNRAS 367, 1417

Rowe, J.F., Matthews, J.M., Kuschnig, R., et al. 2006, Mem.S.A.It. 77, 282

Walker, G.A.H., Matthews, J.M., Kuschnig, R., et al. 2003, PASP 115, 1023 